also advocates the holistic metaphysics of the East and links it with modern physics. Capra, incidentally, quotes approvingly from an important paper of Bohm and Basil Hiley. Capra's book is pleasant to read, for he writes fluently, quotes charming haikus and Zen koans, and even offers some beautiful photographs of sculpture and calligraphy. Bohm's style, by contrast, is ponderous and his exposition abstract. What I find unattractive in Capra's book is the way in which he draws up a brief that "the principal theories and models of modern physics lead to a view of the world which is internally consistent and in perfect harmony with the views of Eastern mysticism". He relies uncritically upon the controversial bootstrap theory of strong interactions, because it fits his metaphysics; he dissolves the precise meaning of technical terms in order to establish analogies; and he plays down discrepancies between points of view when they threaten his parallels. Bohm's book has some of the same tendencies, but to a much lesser degree. Wholeness and the Implicate Order conveys a sense of work in progress, which aims at a distantly glimpsed ideal of the unification of all the aspects of the world, and it is refreshingly free from claims that the ideal has already been achieved. The feeling of struggle in Bohm's book is its most appealing feature.

Abner Shimony is Professor in the Departments of Philosophy and Physics at Boston University.

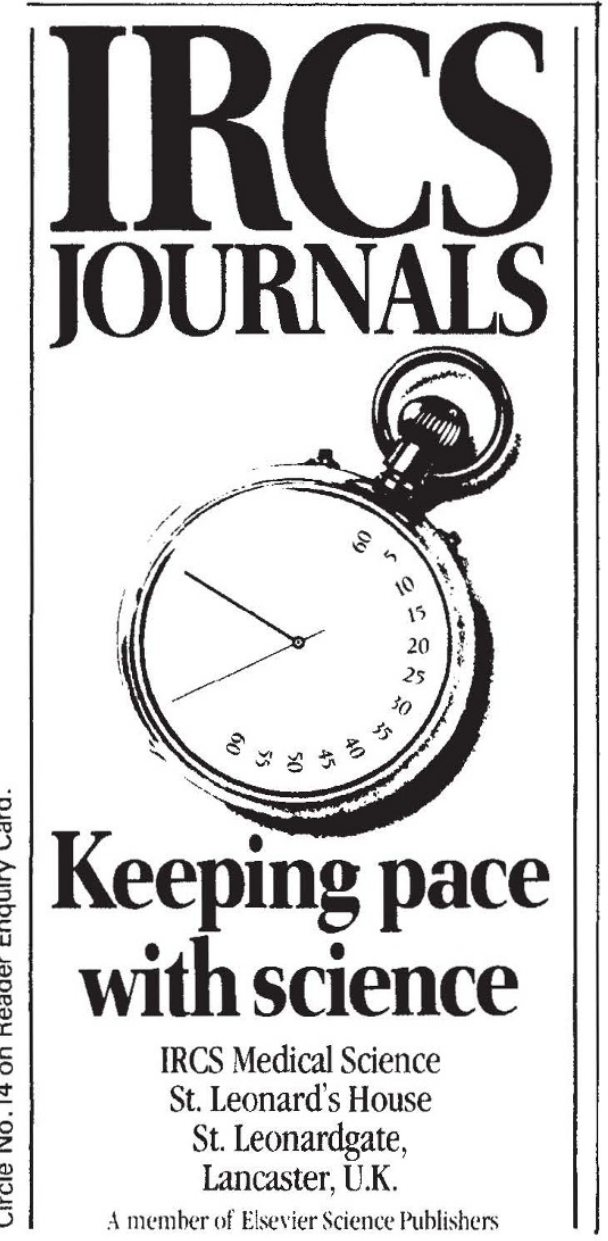

\title{
Birthday party for the skinned fibre
}

\section{C.R. Bagshaw}

Muscle Contraction: Its Regulatory Mechanisms. Edited by S. Ebashi et al. Pp. 549 . ISBN 3-540-10411-9/0-387-10411-9. (Japan Scientific Societies Press, Tokyo/ Springer-Verlag: 1980.) DM108, \$63.80.

DURING October 1979 an international symposium on muscle contraction was held near Mount Fuji in honour of two eminent Japanese scientists, Professor Hiroshi Kumagi and Professor Reiji Natori. In 1955 Kumagi and colleagues identified the "relaxing factor" of muscle as a microsomal ATPase - the now-familiar $\mathrm{Ca}^{2+}$ pump of the sarcoplasmic reticulum. Since then he has been a central figure in organizing and stimulating muscle research in Japan. Natori's "skinned fibre" needs little introduction, having provided a unique preparation amenable to combined physiological and biochemical manipulations. These symposium proceedings have been published in commemoration of Professor Kumagi's seventy-fifth and the skinned fibre's formal twenty-fifth birthday.

The majority of the 42 contributions comprise summaries of recent work, but without unnecessary duplication of published data. Instead, many authors have introduced the historical events and intuitions which have guided the direction of their research. A.F. Huxley starts the proceedings with a review that makes the library stacks as enlightening as the current journal shelves. He describes the initial reluctance to accept that relaxing agents work by chelating $\mathrm{Ca}^{2+}-\mathrm{I}$ wonder if we have become too complacent in our use of EGTA. Natori relates how his skinned fibre helped in the evaluation of the excitation-contraction mechanism, but not content to dwell on history he goes on to describe the contribution of connectin to passive elasticity.

The remaining papers range from studies of cross-bridge movement to the cytoskeleton, but predominantly concern regulatory mechanisms. Unfortunately, while the controversies regarding smooth muscle control are outlined, insufficient data are presented to allow a rational assessment. Hartshorne and Adelstein focus on the properties of kinases, while Nonomura's leiotonin data are limited. Watanabe presents the noteworthy result that desensitized molluscan actomyosin does not superprecipitate, yet SzentGyörgyi shows that desensitized fibres contract regardless of $\mathrm{Ca}^{2+}$. Over the past decade superprecipitation has fallen out of favour as a quantitative probe; clearly, caution is required even in its qualitative applications, particularly in preparations which may possess atypical solubility at low ionic strength.

This volume has achieved its aim of bringing together current research on regulatory mechanisms. The readable styles adopted by most authors serve to complement the existing literature, and the editors are to be congratulated on compiling a fitting and useful tribute.

C.R. Bagshaw is a Lecturer in Biochemistry at the University of Leicester.

\section{Polymers in the lab}

\section{Herman Mark}

Experimental methods in Polymer Chemistry. By J. F. Rabek. Pp.861. ISBN 0-47127604-9. (Wiley: 1980.) £52.80, \$145.20.

THIS presentation of a large and important field of modern chemistry is truly remarkable, particularly since it has been compiled and written by a single author.

Two chapters are essentially descriptive, as they ought to be - the overviews of the structure (Chapter 1) and of the morphology (Chapter 26) of polymers; both contain well-selected and educationally impressive sketches and photographs of all aspects of their topics. In addition they make excellent reading. The bulk of the book is really a condensed encyclopaedia of all the basic characteristics of synthetic polymers. In each case (for instance light scattering, Chapter 13) there is first a clear yet concise presentation of the theoretical background, then a description of the best existing instruments with adequate instructions for making sample preparations and, finally, the most recent improvements and modifications of the particular method are discussed. This enumeration starts with dissolution and goes over fractionation, osmometry, ultracentrifugation, viscometry, all spectroscopic methods to the scattering of $\mathrm{X}$-rays, electrons and neutrons, to NMR and the various chromatographic techniques.

Deserving of particular mention are the condensed but clear presentations of the principles of every individual technique and the many long and carefully assembled tables on significant results, for example those on solubility parameters, NMR parameters and ESR parameters. A special feature of the book, and at the time proof of the never-ending patience of the author, is the bibliography which takes up almost 150 pages and lists 7,350 entries.

If anybody wants to get - or stay - in close touch with polymer chemistry, he ought to buy this book and carry it with him all the time; there is no better source of information on the subject.

Herman Mark is Emeritus Dean of the Polytechnic Institute of New York, and editor of the Journal of Polymer Science. 